\title{
Twenty four hour manometric recording of colonic motor activity in healthy man
}

\author{
${ }^{*}$ F NARDUCCI, G BASSOTTI, M GABURRI, AND A MORELLI \\ From the Gastro-Intestinal Motility Laboratory, First Department of Internal Medicine, University of \\ Perugia, Perugia, Italy.
}

SUMMARY The motor activity of the transverse, descending, and sigmoid colon was recorded for 24 hours in 14 healthy volunteers with a colonoscope positioned catheter. During the study the patients ate two $1000 \mathrm{kcal}$ mixed meals and one continental breakfast. Colonic motor activity was low before meals and minimal during sleep; the motility index increased significantly after meals and at morning awakening. Most of the motor activity was represented by low amplitude contractions present singly or in bursts, which showed no recognisable pattern. All but two subjects also showed isolated high amplitude (up to $200 \mathrm{mmHg}$ ) contractions that propagated peristaltically over long distances at approximately $1 \mathrm{~cm} / \mathrm{sec}$. Most of these contractions occurred after morning awakening, and some in the late postprandial period, with a mean of $4 \cdot 4 /$ subject/ $24 \mathrm{~h}$. The peristaltic contractions were often felt as an urge to defecate or preceded defecation, and could represent the manometric equivalent of the mass movements.

The physiological motor activity of the colon is less well understood than that of any gastrointestinal viscus $^{12}$ as most studies of colonic motor activity are limited to the rectosigmoid area because of the relative inaccessibility of the colon in vivo, and are generally limited to very short observation periods.

The purpose of the present study was to evaluate the characteristics of the motor activity of the transverse, descending, and sigmoid colon in healthy subjects during a 24 hour recording period.

\section{Methods}

\section{SUBJECTS}

Studies were done in 14 healthy volunteers, (11 men), aged 20-32 years. Written informed consent was obtained from all volunteers. All the subjects had on the average one bowel movement per day and no previous abdominal surgery. All denied having bowel irregularities, abdominal pain, or distention.

Bowel cleansing was done with magnesium sulphate $30 \mathrm{~g}$ administered orally 36 hours before

Address for correspondence: Gabrio Bassotti. MD, Clinica Medica I. Policlinico Monteluce. 16100() Perugia. Italy.

Received for publication 22 April 1986.

*Deceased 8 October, 1985. colonoscopy. After the cathartic the subjects ate semiliquid low residue meals for 24 hours and fasted for the remaining 12 hours before endoscopy. Premedication for colonoscopy consisted of diazepam, $10 \mathrm{mg}$ iv. At $8 \mathrm{am}$ after an overnight fast a multilumen manometric catheter was passed up to the transverse colon with the aid of a colonoscope. The manometric catheter was introduced up to the transverse colon by advancing it together with the colonoscope, the tip of the probe being fixed to the tip of the colonoscope by a silk thread held by biopsy forceps maintained inside the operative channel of the colonoscope. When the hepatic flexure was reached the tip of the catheter was released and the colonoscope gently withdrawn, leaving the catheter in situ. After completion of colonoscopy the subjects remained recumbent or seated until 1130 am when the position of the probe was checked radiologically. The tip of the catheter was observed at the hepatic flexure in 11 subjects; it was near the splenic flexure in the remaining three. The position of the catheter was again checked at the end of the study (total radiation exposure to the gonads: $\simeq 30 \mathrm{mrem})$. No major displacement $(>10 \mathrm{~cm})$ occurred in 11 subjects. In two subjects the time of catheter displacement occurred after defecation and the study was terminated. In another study, major catheter displacement probably occurred during a crampy 
urge to defecate and subsequent recording was not used for the analysis.

The manometric probe was an 8 lumen PVC catheter, external diameter 4.5 and internal diameter $0.8 \mathrm{~mm}$ for each lumen. The lumens terminated distally with side openings spaced $12 \mathrm{~cm}$ apart. For each study four consecutive recording sites were selected from the transverse and descending (seven studies), or from the descending and sigmoid colon (seven studies). Intraluminal pressures were recorded by external physiological pressure transducers (Bell \& Howell, type 4-327-1) coupled to a four channel Beckman R-611 Dynograph recorder. The lumens were constantly perfused with bubble free distilled water at $0.1 \mathrm{ml} / \mathrm{min}$ (total volume perfused during 24 hours $\simeq 576 \mathrm{ml}$ ) by a low compliance perfusion system (Arndorfer Medical Specialties). At this perfusion rate, the system yields a pressure rise to occlusion of more than $130 \mathrm{mmHg} / \mathrm{sec}$.

The manometric recordings began at 12 noon and were continued for 24 hours with the subjects lying on a comfortable bed in a quiet room. After a two hour fast the subjects assumed a semirecumbent position and a $1000 \mathrm{kcal}$ mixed lunch was served at $2 \mathrm{pm}$. The meal consisted of a white bread $(46 \mathrm{~g})$ sandwich, with roast beef $(132 \mathrm{~g})$ and mayonnaise $(22 \mathrm{~g})$ and a milkshake $(150 \mathrm{~g})$ with vanilla ice cream $(132 \mathrm{~g})$ : this meal has been previously shown to increase colonic motility. ${ }^{34}$ All the subjects ate the meal in approximately 15 minutes. The subjects remained recumbent thereafter all afternoon and were allowed to read, listen to music, or watch television. Four subjects slept for 30 to 50 minutes at different times during the afternoon. At $8 \mathrm{pm}$ the subjects were given an identical meal. During the night all the subjects slept spontaneously. At 7 am they were awakened (two awoke spontaneously before $7 \mathrm{am}$ ) and all were asked to assume a seated position, for recording the effects on body position changes. One hour later $(8 \mathrm{am})$ a continental breakfast (two croissants and one cup of milk with sugar, total calories $=420$ ), was served.

Throughout the study, the subjects were asked to signal with a manual marker an urge to defecate; a bed pan was provided when necessary. Although most subjects felt an urge to defecate during the study, only two actually did so.

ANALYSIS OF DATA

The recordings were analysed visually. A quantitative analysis was carried out by two of the authors ( $\mathrm{GB}$ and $\mathrm{MG}$ ) by calculating the motility index (product of the mean amplitude of pressure waves multiplied by the sum of their duration). Movement artifacts gave rapid fluctuations which occurred simultaneously at all the four recording sites and were easily differentiated from colonic contractions. Intraluminal basal pressure was taken as baseline (zero reference). Contraction amplitude was calculated by subtracting mean resting colonic pressure from the peak of the pressure wave. Velocity of peristalsis was calculated from the time between peristaltic wave peaks from adjacent transducers. Only waves with amplitude $>20$ $\mathrm{mmHg}$ were analysed. When variations or shift of baseline occurred, this was promptly recognised.

A qualitative analysis was then carried out by three of the authors (FN, GB, MG), independently several times by each one. Some kinds of motor activity were recognised by all the three observers (see Results).

Statistical analysis was done using the paired Student's $t$ test. Unless otherwise specified, results are presented as mean \pm SEM.

\section{Results}

QUANTITATIVE ANALYSIS

The motility index of the transverse, descending and sigmoid colon fluctuated widely during the study (Fig. 1). At all the colonic levels the motility index was low before lunch and dinner, and minimal during the night. The index increased significantly after eating and after the morning awakening. No significant differences were found in the response to the two meals. During the night (arbitrarily defined as the time between $10 \mathrm{pm}$ and $6 \mathrm{am}$ ), periods of complete motor quiescence (absence of contractions at all the recording points) were significantly longer than during a preceding period (from $2 \mathrm{pm}$ to $10 \mathrm{pm}$ ) at all the three colonic segments (Fig. 2). There were no significant differences between the transverse, descending, and sigmoid colon with respect to the motility index and periods of motor quiescence.

QUALITATIVE ANALYSIS

Periods of complete motor quiescence irregularly alternated with periods of motor activity. Phases of motor quiescence lasted up to two hours and were interrupted by little motor activity during the night, when the subjects slept. Waking for a short time during the night generally replaced motor quiescence by activity. Motor quiescence was also predominant during the occasional daytime naps and awakening was promptly accompanied by activity. Infrequent and very short phases of motor quiescence were observed in the early postprandial period and after morning awakening.

With rare exceptions (see below), the large bowel motor activity was characterised by contractions of 


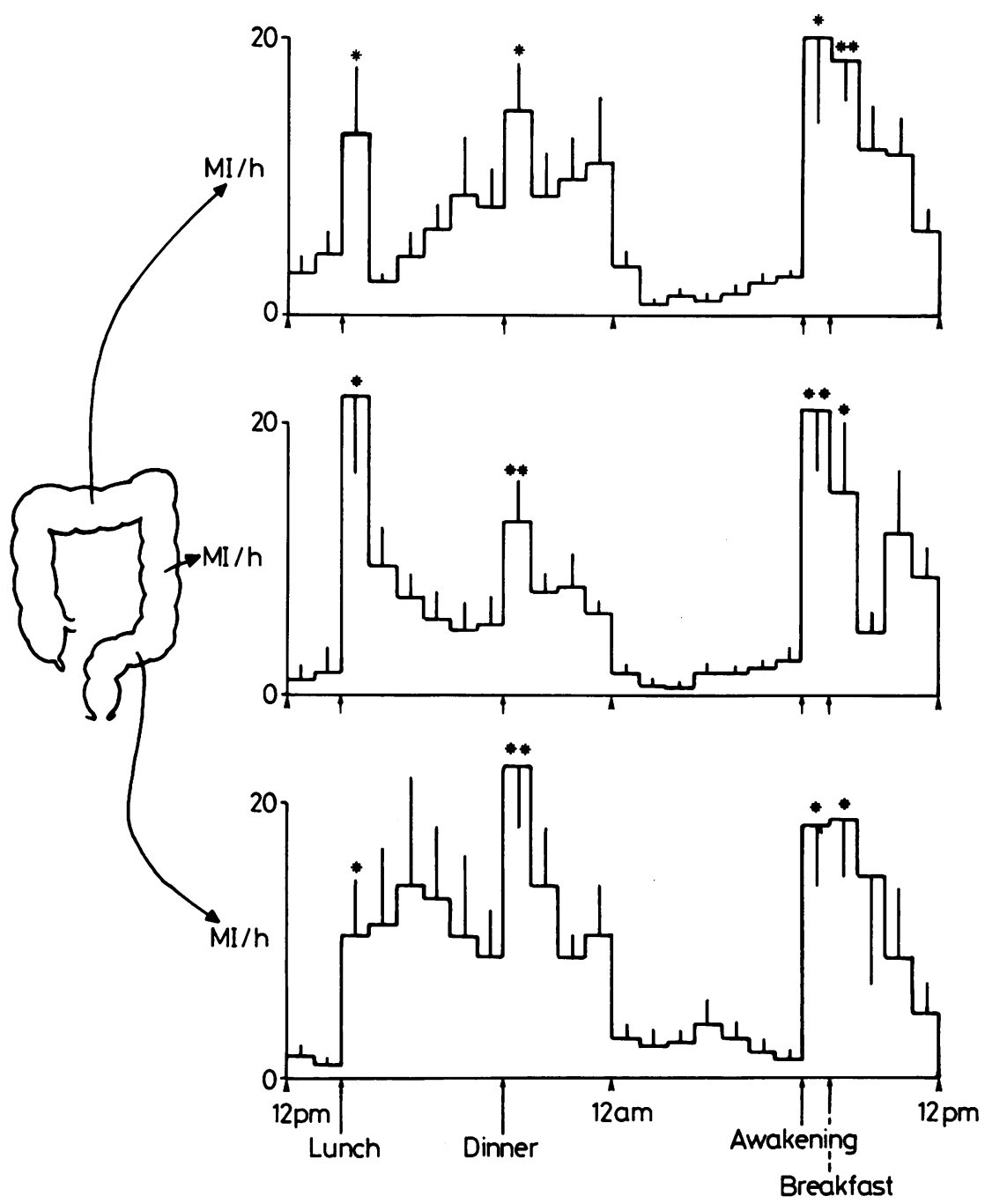

Fig. 1 Motility index/hour $\left(M I / H, \times 10^{-3}\right.$ ) of the transverse (upper diagram), descending (mid), and sigmoid (lower) colon during the 24 hour recording period. Values are expressed as mean $\pm S E M$. Asterisks indicate significant differences $\left({ }^{*} p<0.05,{ }^{* *} p<0.01\right)$ in respect to the fasting and the preawakening values. Abbreviations: $12 p m=12$ noon; $12 a m=12$ midnight.

relatively low amplitude $(\leq 60 \mathrm{mmHg})$. These contractions occurred sometimes sporadically, sometimes in bursts. The low amplitude sporadic contractions occurred at one (Fig. 3) or more recording sites. When appearing at more than one recording site, they usually occurred independently, showing no progressive propagation between them (sporadic non-propagating contractions). Occasionally, however, one of these low amplitude contractions appeared at one recording site a few seconds after a similar contraction at the next (oral or aboral) recording site. Some of these could possibly represent waves cephalad or aborad propagated, over short distances: they never reached a third recording site.

Most of the low amplitude contractions appeared in bursts. In each burst, the contractions occurred either rhythmically most often at a frequency of approximately three/min (Fig. 4), or at six to eight/min (Fig. 5), or arrhythmically. Some of the bursts were present only at one level, with the other recording sites inactive. At other times, especially in 

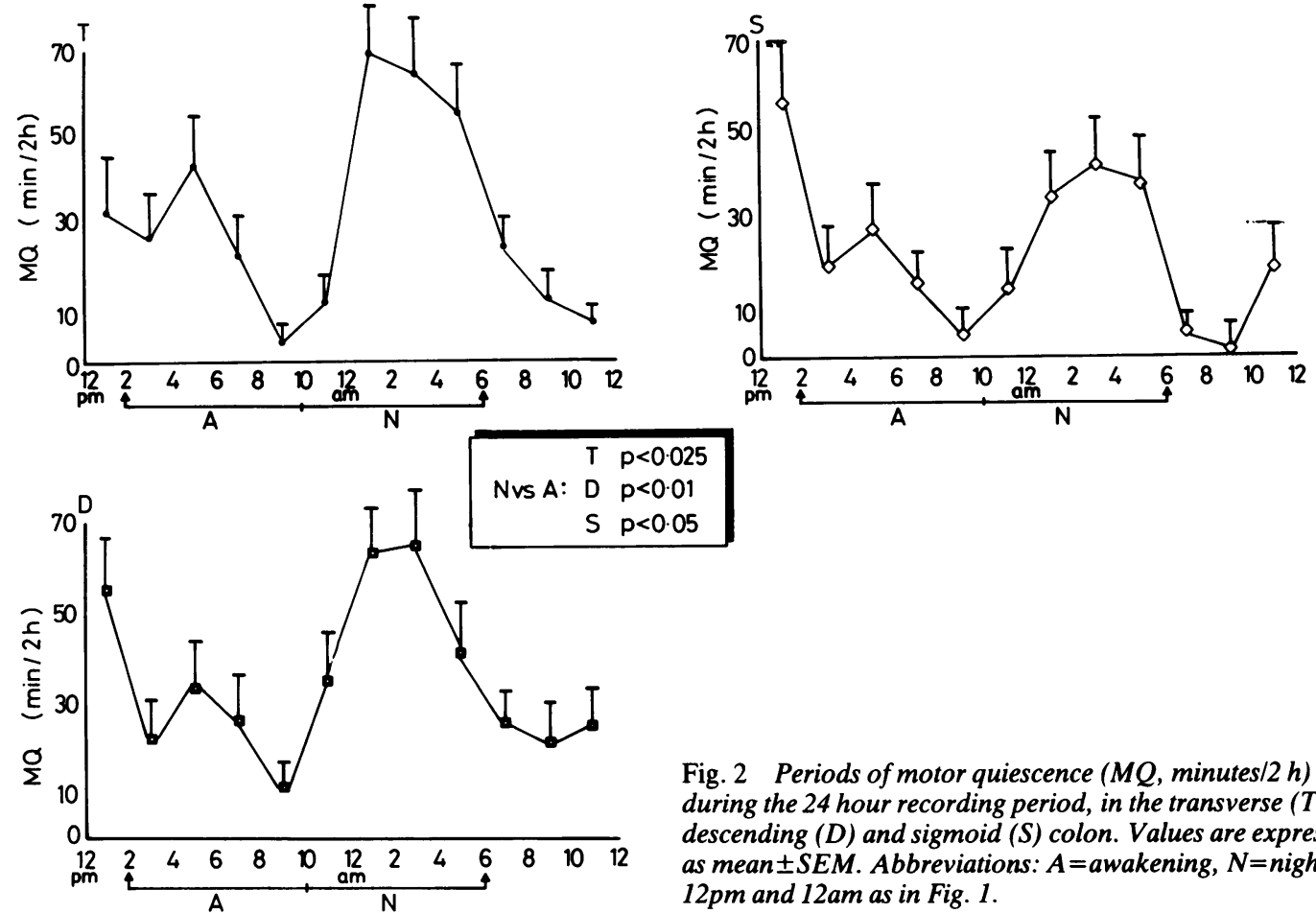

Fig. 2 Periods of motor quiescence (MQ, minutes $/ 2 h)$ during the 24 hour recording period, in the transverse $(T)$, descending $(D)$ and sigmoid $(S)$ colon. Values are expressed as mean \pm SEM. Abbreviations: $A=$ awakening, $N=n i g h t$, 12pm and 12am as in Fig. 1.

Fig. 3 Example of a sporadic non-propagating contraction. 

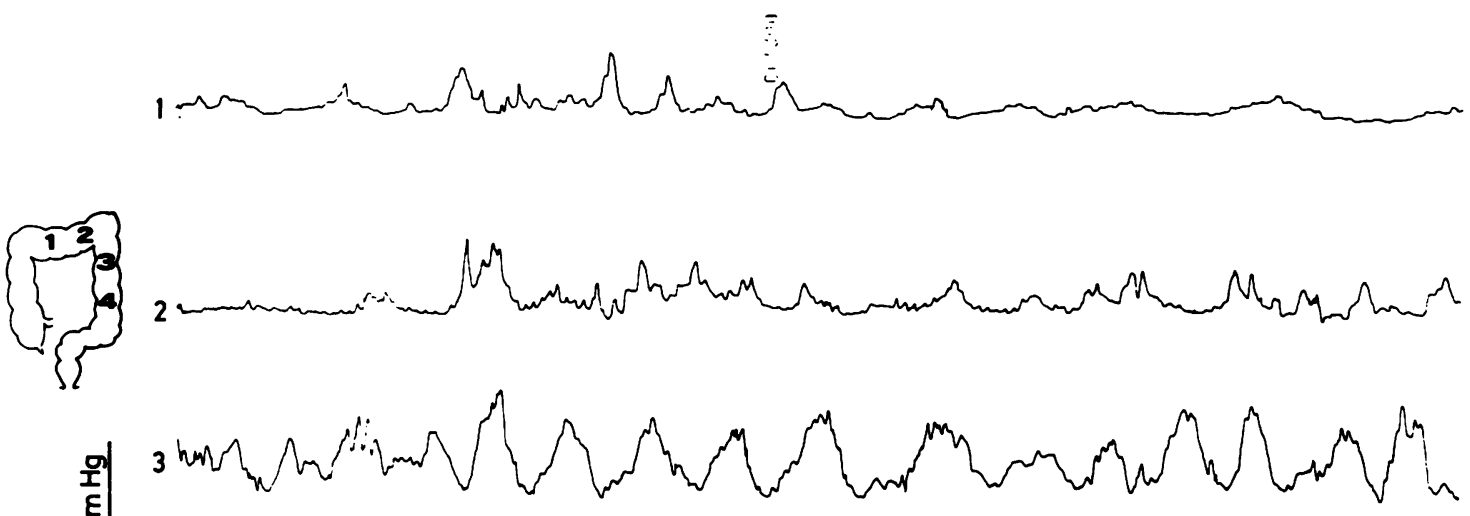

30 sec

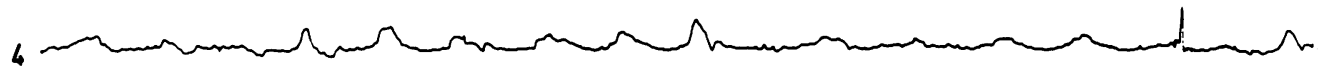

Fig. 4 Example of a non-propagating burst of contractions at approximately 3 cycles/minute.
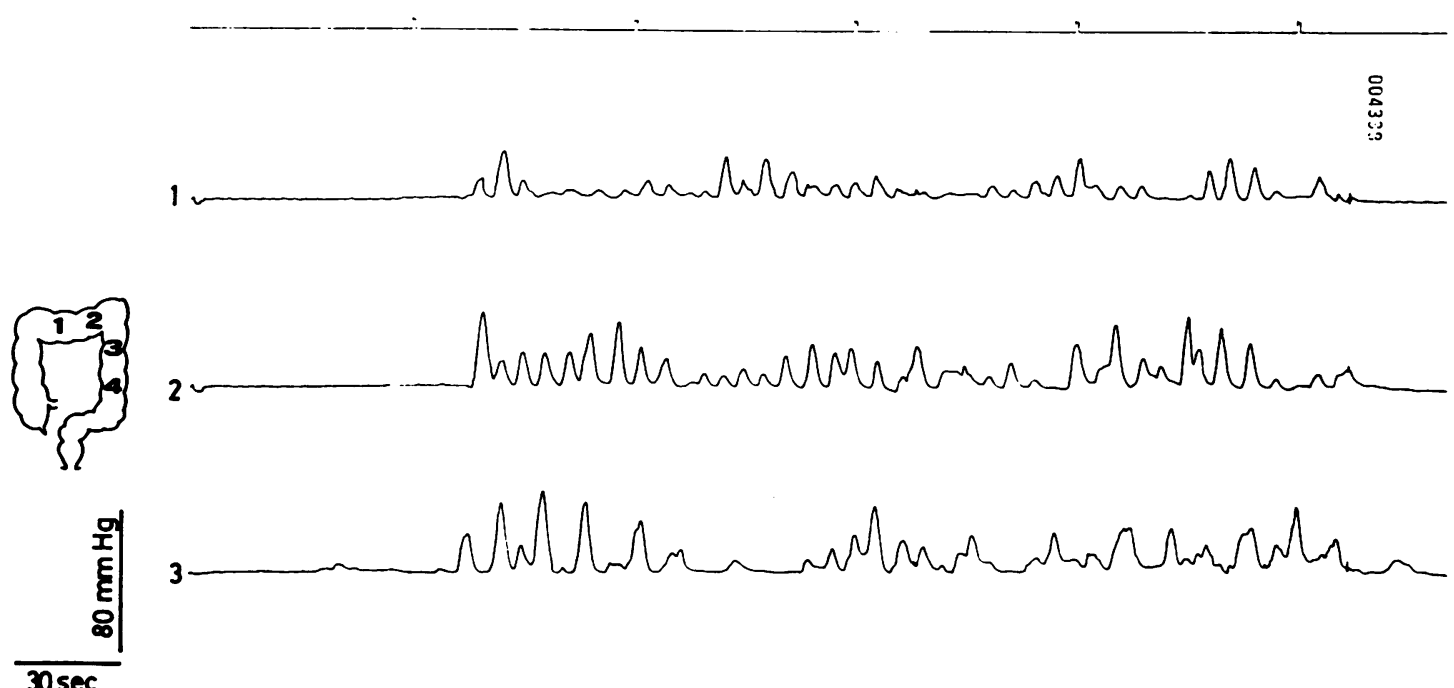

Fig. 5 Example of a non-propagating burst of contractions at approximately 8 cycles/minute.

the early postprandial period, two or more consecutive or distant sites were simultaneously or nearly simultaneously occupied by bursts of contractions. None of these bursts of contractions migrated sequentially, however, orad or aborad through all the recording points. Apart from their greater frequency and duration (up to 20 minutes) after eating and awakening, the occurrence of nonpropagated bursts of contractions showed no recognisable pattern. It is noteworthy that none of the three independent observers was able to identify any cyclic motor pattern such as described by Sarna 


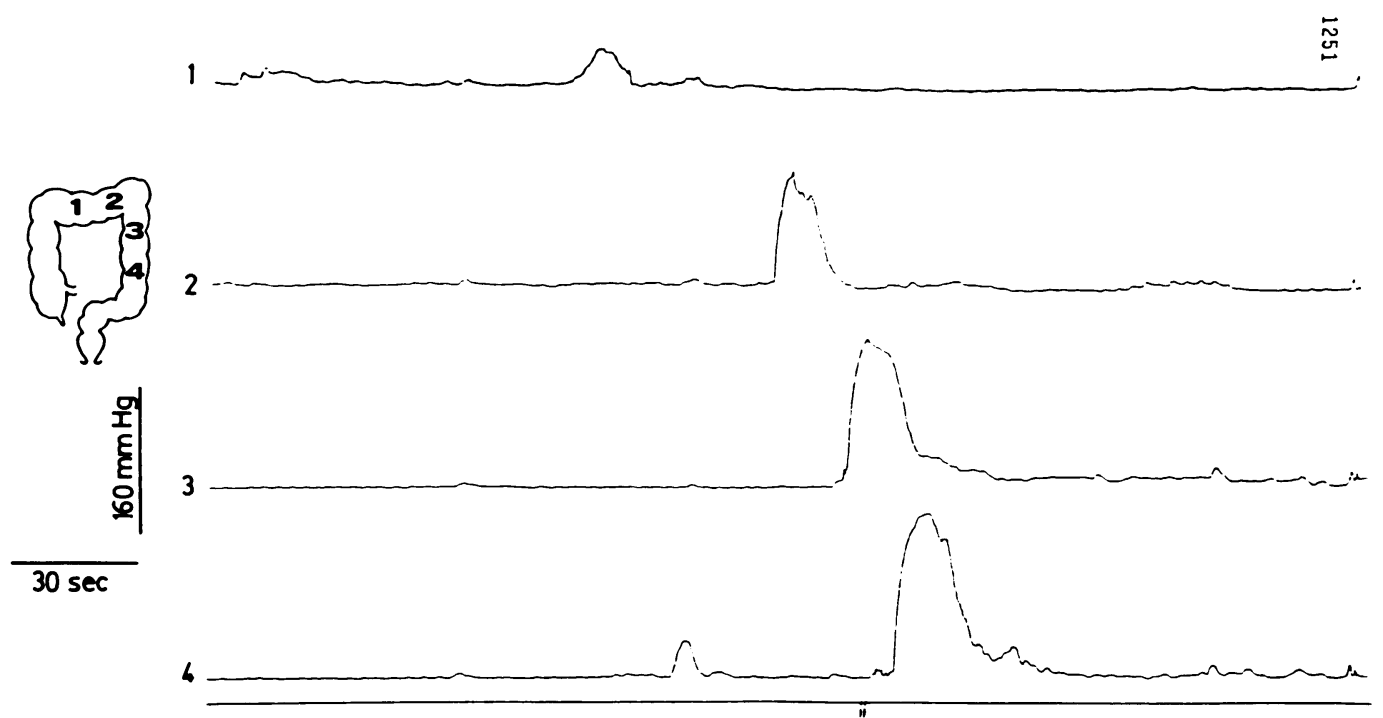

Fig. 6 Example of an high-amplitude propagated contraction (HAPC). Note that in correspondence of the peristaltic contraction the subject signals that he feels an urge to defecate (lower trace).

et $a l^{5}$ in the dog. Contractions of very high amplitude (100-200 $\mathrm{mmHg}$ ) occurred infrequently. The appearance of one high amplitude contraction at one site was always followed some seconds later by a similar contraction at the next and then at a third aboral recording site (Fig. 6). All the high amplitude contractions were propagated peristaltically and termed high amplitude propagated contractions, or HAPC. None of these contractions propagated antiperistaltically.

The visual identification of HAPC was always easy, given their amplitude which did not overlap with the other contractions and their unequivocal propagation over at least three recording sites. Their mean amplitude was $158 \pm 21 \mathrm{mmHg}$ and the mean propagation velocity was $0.9 \pm 0.4 \mathrm{~cm} / \mathrm{sec}$. The origin of HAPC was generally in the transverse colon (transverse and descending colon recordings) and often they propagated to the proximal, or even the distal sigmoid colon (descending and sigmoid colon recordings). Occasionally one HAPC was followed one or two minutes later by a second and occasionally by a third HAPC. When recorded during a period of non-propagated bursts of contractions, a short phase of inhibition of the nonpropagated burst occurred immediately before the HAPC.

High amplitude propagated contractions were recorded in all but two subjects during the study. Their mean frequency was of 4.4 HAPC/subject/ 24 hours. Most of the HAPC occurred after the morning awakening, but some also occurred in the late postprandial period (Fig. 7). When a HAPC appeared every subject experienced a mild abdominal discomfort or a desire to defecate stimulus. Twenty five per cent of the HAPCs were felt as an urge to defecate, and two subjects defecated immediately after HAPC (Fig. 8).

\section{Discussion}

The colon is a neglected viscus concerning studies of its motor activity proximal to the rectosigmoid. With

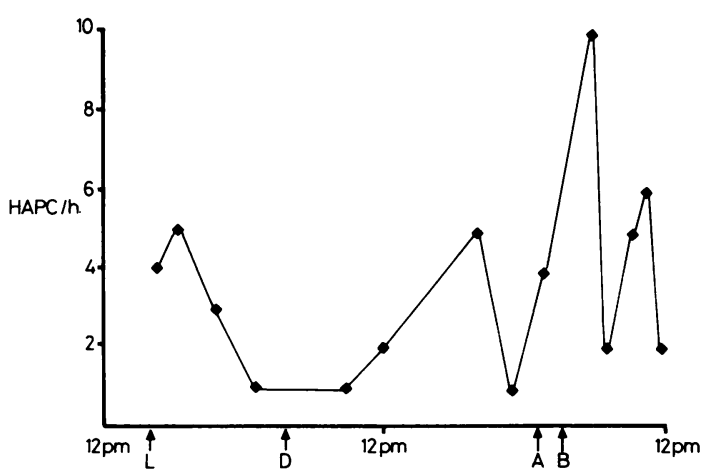

Fig. 7 Total distribution of HAPC over 24 hours. Abbreviations: $A=$ awakening, $B=$ breakfast,$L=l u n c h$, $D=$ dinner, $12 \mathrm{am}$ and $12 \mathrm{pm}$ as in figure $I$. 


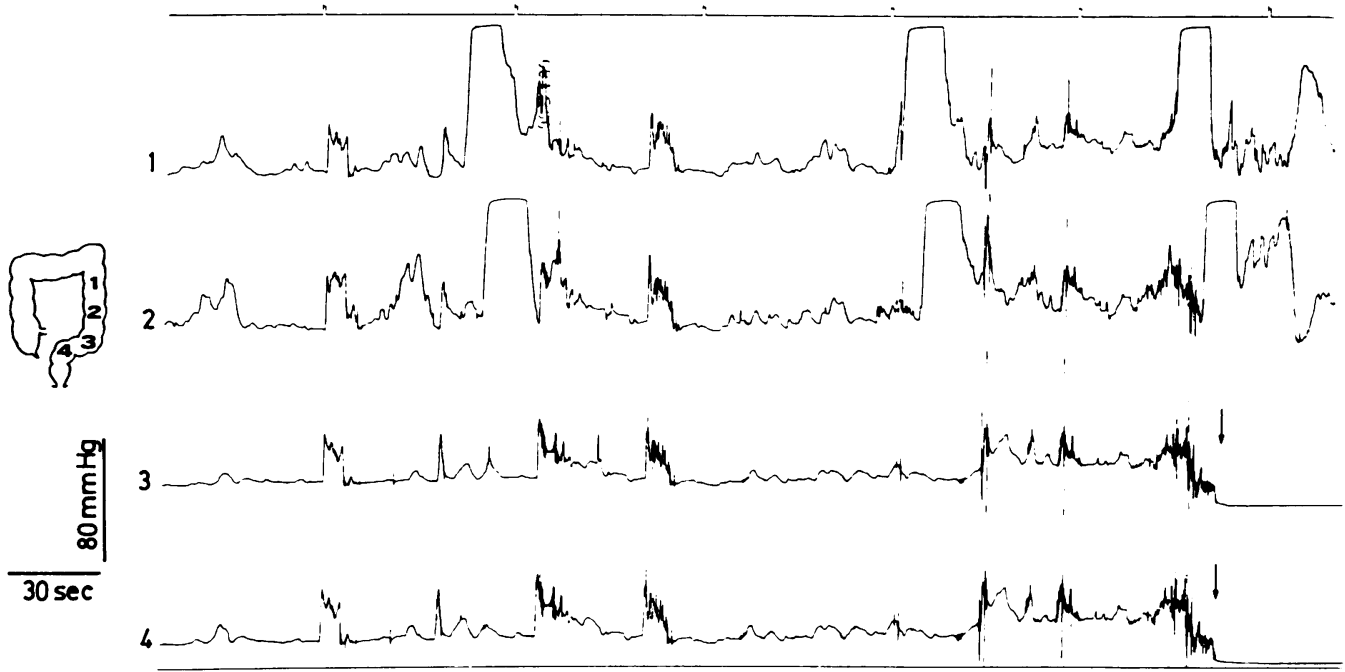

Fig. 8 Colonic motility during defecation. In this case the subject defecated in concomitance with the third HAPC; note the catheter is partially expelled with defecation (arrows).

the recording technique used in this study data were obtained concerning the motor activity of the transverse, descending and sigmoid colon.

Although care was taken to minimise interference with colonic motility by preparation for colonoscopy, it is likely that physiological basal conditions were not present, because the colons were empty and the subjects ate low residue meals. Secondly, colonic motor activity was recorded with the subjects recumbent throughout the study. These conditions must be taken into account and suggest caution in extrapolation of these results.

Despite some criticism, ${ }^{26}$ the use of open ended catheters perfused by low compliance infusion systems is a useful tool for recording intraluminal pressures from the small and the large bowel. ${ }^{78}$ Whether it records all, or only part of contractile activity remains to be determined.

In this study colonic motor activity recorded over 24 hours was characterised by irregular alternation of quiescences, sporadic non-propagating contractions, non-propagating bursts of contractions and high amplitude propagated contractions (HAPC). Regular cyclic motor activity was not observed. Unlike in the small bowel, the alternation of phases of motor quiescence with activity in fasting and fed states was irregular and unpredictable, suggesting that colonic motility is not governed by a rhythmic interdigestive pattern. A similar distribution of motor activity has been reported previously in the distal colon of healthy subjects. ${ }^{79}$ 10

At all the three colonic segments investigated motor activity was chiefly represented by low amplitude, non-propulsive segmental contractions, while peristalsis, although vigorous, occurred seldom. These findings are similar to those found in early investigations performed using time lapse cinematography. Ritchie ${ }^{11}$ observed segmental contractions capable of pushing contents either antegrade or retrograde, over short distances. These segmental contractions might slow colonic transit. We hypothesise that the prevalent non-propulsive motor activity: the non-propagated sporadic and bursts of contractions might be the manometric equivalent of segmental contractions observed by cineradiography.

Radiological studies also suggested that colonic contents can be transported in a retrograde direction. ${ }^{12} 13$ Contractions that unequivocally propagated in a retrograde direction were not recorded in this study, although as recording points were $12 \mathrm{~cm}$ apart, it cannot be excluded that some of the low amplitude sporadic contractions propagated either orally or aborally over short distances. Retrograde propulsion in the colon does not require retrograde peristalsis, as contents can be squeezed orally or aborally by segmental contractions. This study provides insufficient data pertaining to the ascending colon, where retrograde propulsion is believed to be prevalent. ${ }^{2}$

Up to now colonic peristalsis has been very poorly documented. Early radiological studies showed that colonic contents are infrequently propelled by mass movements over long colonic segments at approximately $0.5 \mathrm{~cm}$ sec approximately three or four times per day. ${ }^{14-16}$ Direct evidence of colonic 
peristalsis was obtained in two early studies, where the colon proximal to the rectosigmoid was reached through colostomies ${ }^{17}$ or with an end-to-end pilot intubation. ${ }^{18}$ In both studies the observation period was not long enough to show spontaneous peristalsis, but peristalsis was recorded after stimulation with contact laxatives, the peristaltic contractions being of high amplitude and propagated distally at 0.5 $\mathrm{cm} / \mathrm{sec}$. Torsoli et al using a combined manometric and radiological technique demonstrated the correlation of these high amplitude contractions with mass movements stimulated by laxatives. ${ }^{18}$

In the present study unequivocal spontaneous peristaltic activity was recorded from the transverse, descending and sigmoid colon. These peristaltic contractions were always easily differentiated on visual analysis from other contractions because of their unique characteristics, which comprise high amplitude and unequivocal propagation over long distances. The high amplitude propagated contractions had characteristics strikingly similar to those reported in older radiological studies reporting mass movements. The peristaltic activity was rare, with a mean frequency of four per subject per $24 \mathrm{~h}$, and a propagation velocity of $1 \mathrm{~cm} / \mathrm{sec}$; they always travelled towards the anus. The high amplitude peristaltic contractions were often associated with an urge to defecate or preceded defecation. It is therefore probable that high amplitude peristaltic contractions are the manometric equivalent of mass movements.

During the 24 hours colonic motility fluctuated widely and these fluctuations were mainly related to the fasting/eating or sleeping/awakening periods. As in previous studies, ${ }^{3419}$ the motor activity of the distal colon promptly increased in response to high caloric mixed meals. The present data show that a similar response to eating also occurs in the descending and transverse colon. This response was characterised by non-propagating motor activity, while peristaltic contractions appeared a few hours after eating and after meal related colonic stimulation had waned. These late postprandial high amplitude propagated contractions might be promoted by the colonic distention because of the arrival new faeces in the colon. ${ }^{20}$ We have recently reported that colonic distention with a balloon can promote high amplitude propagated contractions. ${ }^{21}$

The low caloric breakfast was also followed by enhanced motor activity but this response should be interpreted with caution as the recent stimulus produced by morning awakening might not have terminated at that time.

Finally, sleep was nearly always associated with a sharp inhibition of colonic motor activity (Figs 1 and 2). Similarly, an early study reported an inhibition of colonic motility during drug induced naps, while the following awakenings were associated with enhanced motor activity. ${ }^{22}$

In summary, colonic motor activity is mainly represented by non-propagating contractions, occurring either sporadically or in bursts; colonic peristalsis is infrequent, but vigorous, and has characteristics strongly suggesting it could be the manometric equivalent of the mass movements; eating and morning awakening are major stimuli in eliciting colonic motility.

We are indebted to Professor E E Daniel for reviewing the manuscript and useful suggestions, Ms Michele Kildea for reviewing the English and particularly to Dr Pierluca Narducci and Mrs Francesca Narducci for encouragement.

\section{References}

1 Misiewicz JJ. Colonic motility. Gut 1975; 16: 311-4.

2 Christensen J. Motility of the colon.In Johnson R, ed. Physiology of the gastrointestinal tract. New York: Raven Press, 1981.

3 Sullivan MA, Cohen S, Snape WJ. Colonic myoelectrical activity in irritable bowel syndrome. Effect of eating and anticholinergics. $N$ Engl J Med 1978; 298: 878-83.

4 Narducci F, Bassotti G, Granata MT, et al. Colonic motility and gastric emptying in patients with irritable bowel syndrome. Effect of pretreatment with octylonium bromide. Dig Dis Sci 1986; 31: 241-6.

5 Sarna SK, Condon R, Cowles V. Colonic migrating and non migrating motor complexes in dogs. Am J Physiol 1984; 246: G355-G60.

6 Wingate DL. Methodology of motility. In: Christensen J, Wingate DL, eds. A guide to gastrointestinal motility. Bristol: Wright, 1983.

7 Kerlin PL, Zinsmeister A, Phillips S. Motor responses to food of the ileum, proximal colon and distal colon of healthy humans. Gastroenterology 1983; 84: 762-70.

8 Campbell D, Shannon S, Reddy N, Valori R, Collins SM: The fidelity of recording techniques and intrasubject variability in human colonic motility studies. Gastroenterology 1985; 88: 1341.

9 Narducci F, Bassotti G, Pelli MA, Morelli A. Prolonged recording of myoelectric and contractile activities of the human colon. Ital J Gastroenterol 1984; 16: 317-35.

10 Dinoso VP, Murthy SNS, Goldstein J, Rossner B. Basal motor activity of the distal colon: a reappraisal. Gastroenterology 1983; 85: 637-42.

11 Ritchie JA. Movements of segmental constrictions in the human colon. Gut 1971; 12: 350-5.

12 Ritchie JA. Colonic motor activity and bowel function. Part II: distribution and incidence of motor activity at rest and after food and carbachol. Gut 1968; 9: 502-11. 
13 Ritchie JA, Truelove SC, Ardran GN, Tuckey MS. Propulsion and retropulsion of normal colonic content. Dig Dis Sci 1971; 8: 697-703.

14 Holzknecht G. Die normale peristaltik des colon. Munch Med Wochenschr 1909: 56: 2401-3.

15 Hertz AF, Newton A. The normal movements of the colon in man. $J$ Physiol (Lond) $1913 ;$ 47: 57-65.

16 Ritchie JA. Mass peristalsis in the human colon after contact with oxyphenisatin. Gut 1972; 13: 211-19.

17 Hardcastle JD, Mann CV. Study of the large bowel peristalsis. Gut 1968; 9: 512-20.

18 Torsoli A, Ramorino ML, Ammaturo MV, Capurso L, Paoluzi P, Anzini F. Mass movements and intracolonic pressures. Am J Dig Dis 1971; 16: 693-6.
19 Narducci F, Bassotti G, Daniotti S, Del Soldato P, Pelli MA, Morelli A. Identification of muscarinic receptor subtype mediating colonic response to eating. Dig Dis Sci 1985; 30: 124-8.

20 Kerlin P, Phillips S. Differential transit of liquids and solids through the human ileum. Am J Physiol 1983; 245: G38-G43.

21 Narducci F, Bassotti G, Gaburri M, Solinas A, Fiorucci S, Morelli A. Distention stimulated motor activity of the human transverse, descending and sigmoid colon. Gastroenterology 1985; 88: 1515.

22 Rosemblum MJ, Cummings AJ. The effect of sleep and of amytal on the motor activity of the human sigmoid colon. Gastroenterology 1954; 27: 445-50. 